Dear author,

Please note that changes made in the online proofing system will be added to the article before publication but are not reflected in this PDF.

We also ask that this file not be used for submitting corrections. 


\title{
Analytical bounds for the electromechanical buckling of a compressed nanocantilever
}

\author{
Enrico Radi*, Giovanni Bianchi, Lorenzo di Ruvo \\ Dipartimento di Scienze e Metodi dell'Ingegneria, Università di Modena e Reggio Emilia, via G. Amendola 2, 42122 Reggio Emilia, Italy
}

\section{A R T I C L E I N F O}

\section{Article history:}

Received 29 August 2017

Revised 20 January 2018

Accepted 12 February 2018

Available online $\mathrm{xxx}$

\section{Keywords:}

Pull-in voltage

MEMS

Q2 NEMS

Nanocantilever

Casimir force

Van der Waals force

Buckling load

\begin{abstract}
A B S T R A C T
An analytical approach is presented for the accurate definition of lower and upper bounds for the pull-in voltage and tip displacement of a micro- or nanocantilever beam subject to compressive axial load, electrostatic actuation and intermolecular surface forces. The problem is formulated as a nonlinear two-point boundary value problem and has been transformed into an equivalent nonlinear integral equation. Initially, new analytical estimates are found for the beam deflection, which are then employed for assessing novel and accurate bounds from both sides for the pull-in parameters, taking into account for the effects of the compressive axial load. The analytical predictions are found to closely agree with the numerical results provided by the shooting method. The effects of surface elasticity and residual stresses, which are of significant importance when the physical dimensions of structures descend to nanosize, can also be included in the proposed approach.
\end{abstract}

(c) 2018 Published by Elsevier Inc.

\section{Introduction}

Several MEMS and NEMS current applications in sensors, actuators and memory devices [1,2] exploit the switching between two stable positions of a flexible electrode nanobeam suspended above a fixed electrode. Under the action of the electrostatic force the nanocantilever deflects toward to the ground and the electrostatic force increases correspondingly. At the pull-in voltage the micro-beam leaves the principal equilibrium path, which becomes unstable, and pulls-in onto the fixed electrode, thus creating an electrical connection. Since the pull-in voltage determines operation voltage and power dissipation, its accurate determination becomes of the primary importance in MEMS and NEMS design. On one side, a low pull-in voltage implies a limited power consumption and small amount of energy stored in the system, thus enhancing the device performance [1]. A method for reducing the pull-in voltage consists in applying a compressive axial load to the nanobeam. On the other side, if the pull-in voltage is too small, the intermolecular surface forces may cause the collapse of the nanocantilever tip onto the fixed electrode, even in the absence of electrostatic actuation. These quantum mechanical interactions are usually described by van der Waals (vdW) or Casimir forces according to the gap between the electrodes [3]. Their effects are negligible for MEMS, where the separation distance is of the order of micron, but they play a significant role for NEMS, where the gap descends to the nanoscale.

The cantilever beam deflection is described by a nonlinear fourth-order boundary value problem (BVP) that can be solved by using approximate approaches only. The most exploited ones are based on the assumption of 1D lumped models or specific shape functions for the beam deflection. Perturbation approaches or Taylor series expansions of the loading term

\footnotetext{
* Corresponding author.

E-mail addresses: eradi@unimore.it, eradi@libero.it (E. Radi).
} 
have been frequently employed also. Detailed information can be found in review papers by Lin and Zhao [4], Chuang et al. [5], Zhang et al. [6]. However, these approximated methods provide random estimates of the pull-in parameters and their accuracy considerably decreases as the actuation voltage gets closer to the pull-in voltage. In order to be effective, the approach should instead offer accurate lower and upper bounds, both for the pull-in voltage and tip deflection.

According to the theory of elastic stability, slender beams have critical compressive load limits beyond which they buckle [7]. If they are also subject to electrostatic loading, their deflection increases nonlinearly with the magnitude of the compressive axial load. Therefore, the determination of the electromechanical buckling (EMB) characteristics of axially-loaded nanobeams is essential for designing such devices [8-12]. Indeed, the application of an adaptable compressive load, e.g. by means of an axial elastic constraint applied at the tip of the beam, allows to modify the deflection and the pull-in behavior of a nanocantilever. It may also affect the occurring of stiction [13-15], namely the phenomenon that takes place when the intermolecular surface forces overcome the restoring elastic forces and keep the cantilever tip attached to the ground. In general, stiction may be avoided or favoured by reducing or increasing the axial compressive load, respectively. Stiction has been advantageously exploited in applications such as non-volatile memory cells, since it allows holding the switch in the closed state with no need for supplying continued power input. On the contrary, in sensor and actuator applications it may cause permanent adhesion and other unexpected occurrences that may reduce the range of operability of the device.

The axial load provides an additional linear second-order term in the governing fourth-order ODE of the Eulero-Bernoulli (EB) beam model, which can either stiffen, if tensile, or soften, if compressive, the nanostructure. Many authors investigated the influence of the axial load and, in general, of the additional second-order term on the beam pull-in displacement and voltage by using a numerical approach [3,16-20]. They clearly found that critical pull-in voltage decreases under the action of a compressive axial load, whereas an opposite stiffening effect is observed for a tensile axial load. Therefore, the presence of a compressive axial load is an important issue in the fabrication and design of sensors and actuators, because it may cause degradation or even failure of the devices.

The work [21] focuses on the effects of the support flexibility on the pull-in instability of an electrostatically actuated micro- or nanocantilever and accurate analytical bounds are found for the pull-in parameter in the absence of axial load. The present work focuses instead on the effects of a compressive axial load on the pull-in instability. To this aim, the analytical approach proposed in [21] is extended by taking into consideration an additional term in the governing ODE and in the boundary conditions coming from the contribution of the axial load. This contribution is indeed expected to have a significant influence on the pull-in parameters [3,22] as well as on the critical gap for a freestanding compressed nanocantilever, which is an essential parameter in the design of NEMS for avoiding the collapse of the flexible electrode on the ground plane when the electrostatic loading is removed. Moreover, the additional term may also include the effects of surface elasticity and residual stresses, which are important factors that may explain the experimentally measured size dependent behavior of nanobeams.

The nonlinear BVP governing the beam deflection is presented in Section 2 and then transformed into an equivalent nonlinear integral equation by using the Green's function of the compressed cantilever beam. Here, we consider a linear elastic EB beam subject to a distributed load that depends nonlinearly on the beam deflection. The total deflection of the beam is formally given by the sum of all the contributions offered by the load acting on each infinitesimal part of the beam. Therefore, it can be calculated by exploiting the Green's function of the compressed cantilever EB beam. A similar approach has been employed also in the papers [3,21], where the contribution of the axial load has not been introduced. The solution of the extended BVP considered here is then proved to be positive, monotonic and convex and novel lower and upper estimates on the deflection are obtained in Section 3. These estimates are then employed in Section 4 for assessing novel and accurate bounds from both sides for the pull-in in voltage and tip deflection, taking into account for the effects of the compressive axial load. Estimates of the critical level of the intermolecular surface forces causing the pull-in instability in the absence of electrostatic loading are also provided for various values of the axial compressive load. The proposed approach is then validated in Section 5 by comparing the analytical estimates with the numerical solution of the BVP obtained by using the shooting method.

Since no closed form solution can be achieved for the extremely nonlinear BVP governing the pull-in instability of a micro- or nanobeam subject to a compressive axial force, but, to authors' knowledge, only numerical or approximate methods have been proposed in the Literarture - e.g. differential quadrature method, Adomian decomposition method, finite element method and the Galerkin method - then, the analytical bounds provided here can be considered as extremely useful for validating the number of numerical strategies and approximated methods used for approaching this very difficult nonlinear BVP. Moreover, the approach is flexible enough to allow for further generalization aiming to consider more complex interactions arising at the micro- and nanoscale, such as electrochemical and double layer interactions.

\section{Mathematical model}

The problem of an elastic micro- or nanocantilever clamped at one end and subject to electrostatic actuation with the effects of fringing field, vdW or Casimir forces, and compressive axial load $P$, sketched in Fig. 1, can be described by the following fourth-order, non-linear ODE

$$
u^{I V}(x)+k^{2} u^{\prime \prime}(x)=f(u(x)), \quad \text { for } x \in[0,1],
$$




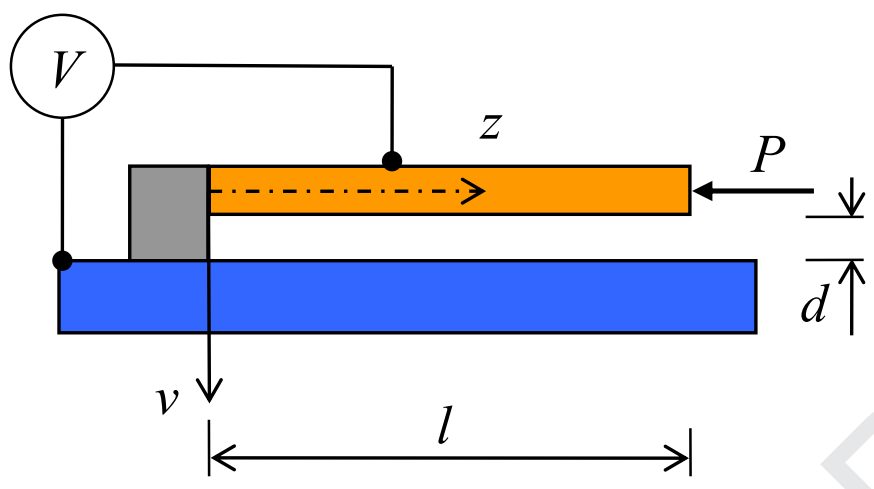

Fig. 1. A compressed micro/nanocantilever under electrostatic actuation.

where $u=v / d$ and $x=z / l$ are nondimensional variables, being $v$ the beam deflection, $d$ the initial gap between the electrodes, $z$ the position along the beam as measured from the clamped end, and $l$ the beam length, so that $0 \leq z \leq l$, and the apex denotes differentiation with respect to $x$. The loading term in (1) includes the contributions of electrostatic actuation, fringing field and van der Waals or Casimir forces, namely

$$
f(u)=\frac{\gamma \beta}{1-u}+\frac{\beta}{(1-u)^{2}}+\frac{\alpha_{W}}{(1-u)^{3}}+\frac{\alpha_{C}}{(1-u)^{4}},
$$

being $\gamma=0.65 \mathrm{~d} / \mathrm{w}$ the fringing coefficient, where $w$ is the cross-section width. The nondimensional parameters $\beta, \alpha_{W}$ and $\alpha_{C}$ are given by

$$
\beta=\frac{\varepsilon_{0} w V^{2} l^{4}}{2 d^{3} E I}, \quad \alpha_{W}=\frac{A w l^{4}}{6 \pi d^{4} E I}, \quad \alpha_{c}=\frac{\pi^{2} h c w l^{4}}{240 d^{5} E I},
$$

where $V$ is the electric voltage applied to the electrodes, $E$ is the Young's modulus of the elastic material and $I$ is the moment of inertia of the beam cross-section, $\varepsilon_{0}=8.854 \times 10^{-12} C^{2} N^{-1} \mathrm{~m}^{-2}$ is the permittivity of vacuum, $A$ is the Hamaker constant, $h=1.055 \times 10^{-34} \mathrm{Js}$ is the Planck's constant divided by $2 \pi, c=2.998 \times 10^{8} \mathrm{~m} / \mathrm{s}$ is the speed of light.

The boundary conditions for the cantilever beam then require vanishing of displacement and rotation of the cross section at $x=0$, and vanishing of the bending moment and shearing force at $x=1$, namely [7]

$$
u(0)=0, \quad u^{\prime}(0)=0, \quad u^{\prime \prime}(1)=0, \quad u^{\prime \prime \prime}(1)+k^{2} u^{\prime}(1)=0,
$$

where the non-dimensional parameter

$$
k=\sqrt{\frac{P l^{2}}{E I}},
$$

denotes the square root of the ratio between the compressive axial load $P$ and the beam bending stiffness. The buckling axial load for a cantilever EB beam is attained as $k$ approaches $\pi / 2$ [7]. Therefore, in the following we assume $0 \leq k<\pi / 2$.

Since the scale effect of the nanostructure is generally compatible with the size of molecular and/or atomic interactions, advanced beam models are usually required for an accurate simulation, such as those derived by the non-local or couple stress elastic theory $[10,11,19,20,23]$. These sophisticated constitutive models contain an internal length scale as a material parameter, which introduce in the governing ODE (1) a further term proportional to the second derivative. The effects of surface tension and residual stresses are also significant in nanobeams. They occur because the physical properties of surface layer are different from that of the bulk of nanoscale material and structure. For conductive metals these effects appear at the submicron scale. They have been simulated in many recent works by using enhanced constitutive models accounting for surface elasticity [24-27].

All these contributions can be included in the present model just by modifying the value of the parameter $k$ and/or introducing additional terms in the flexural stiffness EI of the nanobeam [28-32].

\subsection{Nonlinear integral equation formulation}

The nonlinear BVP (1) and (4) can be equivalently formulated by means of a nonlinear integral equation once the Green's function $G(t)$ of the differential problem is worked out, as done in [21] for a nanocantilever in the absence of the axial load. The deflection of a cantilever EB beam subject to a compressive axial load and a transversal unit load acting at position $x$ is described by the following linear ODE

$$
G^{I V}(t)+k^{2} G^{\prime \prime}(t)=\delta(x-t)
$$


103

where $\delta(x)$ denotes the Dirac delta function. The general solution to the ODE (6) is

$$
G(t)= \begin{cases}A_{0}+A_{1} t+A_{2} \cos k t+A_{3} \sin k t, & 0 \leq t<x, \\ B_{0}+B_{1} t+B_{2} \cos k t+B_{3} \sin k t, & x<t \leq 1 .\end{cases}
$$

The eight constants $A_{i}$ and $B_{i}(i=0,1,2,3)$ can be determined by using the boundary conditions (4), namely

$$
G(0)=0, \quad G^{\prime}(0)=0, \quad G^{\prime \prime}(1)=0, \quad G^{\prime \prime \prime}(1)+k^{2} G^{\prime}(1)=0,
$$

and the continuity conditions at $t=x$ for the deflection, slope, bending moment and shear force

$$
\begin{aligned}
& G\left(x^{+}\right)=G\left(x^{-}\right), \quad G^{\prime}\left(x^{+}\right)=G^{\prime}\left(x^{-}\right), \quad G^{\prime \prime}\left(x^{+}\right)=G^{\prime \prime}\left(x^{-}\right), \\
& G^{\prime \prime \prime}\left(x^{+}\right)+k^{2} G^{\prime}\left(x^{+}\right)-G^{\prime \prime \prime}\left(x^{-}\right)-k^{2} G^{\prime}\left(x^{-}\right)=1,
\end{aligned}
$$

respectively, thus providing

$$
G(t) \begin{cases}\left\{\sin k t-k t+(1-\cos k t)[\sin k x+(1-\cos k x) \tan k\} / k^{3},\right. & 0 \leq t<x, \\ \left\{\sin k x-k x+(1-\cos k x)[\sin k t+(1-\cos k t) \tan k\} / k^{3},\right. & x<t \leq 1 .\end{cases}
$$

Therefore, the BVP (1) and (4) is equivalent to the following non-linear integral equation

$$
\begin{aligned}
u(x)= & \frac{1}{k^{3}} \int_{0}^{x}\{\sin k t-k t+(1-\cos k t)[\sin k x+(1-\cos k x) \tan k]\} f(u(t)) d t \\
& +\frac{1}{k^{3}} \int_{x}^{1}\{\sin k x-k x+(1-\cos k x)[\sin k t+(1-\cos k t) \tan k]\} f(u(t)) d t .
\end{aligned}
$$

As $k \rightarrow 0$, the non-linear integral Eq. (11) recovers that obtained in [3,33,34] for a cantilever EB beam not axially loaded. According to (11), the normalized deflection of the cantilever tip then is given by

$$
u(1)=\frac{1}{k^{3}} \int_{0}^{1}[\sin k t-k t+(1-\cos k t) \tan k] f(u(t)) d t .
$$

By taking the derivatives of Eq. (11) one obtains

$$
\begin{aligned}
u^{\prime}(x)= & \frac{\cos k x+\sin k x \tan k}{k^{2}} \int_{0}^{x}(1-\cos k t) f(u(t)) d t \\
& +\frac{1}{k^{2}} \int_{x}^{1}\{[\sin k t+(1-\cos k t) \tan k] \sin k x+\cos k x-1\} f(u(t)) d t,
\end{aligned}
$$

$$
\begin{aligned}
u^{\prime \prime}(x)= & \frac{\cos k x \tan k-\sin k x}{k} \int_{0}^{x}(1-\cos k t) f(u(t)) d t \\
& +\frac{1}{k} \int_{x}^{1}\{[\sin k t+(1-\cos k t) \tan k] \cos k x-\sin k x\} f(u(t)) d t,
\end{aligned}
$$

$$
\begin{aligned}
u^{\prime \prime \prime}(x)= & -(\cos k x+\sin k x \tan k) \int_{0}^{x}(1-\cos k t) f(u(t)) d t \\
& -\int_{x}^{1}\{[\sin k t+(1-\cos k t) \tan k] \sin k x+\cos k x\} f(u(t)) d t,
\end{aligned}
$$

and, thus, the following lemma holds true.

Lemma 2.1. Let $u(x)$ be the solution to the nonlinear integral Eq. (11), then the following conditions hold for $f(u) \geq 0, k \in[0$, $\pi / 2)$ and $x \in[0,1]$ :

$$
u(x) \geq 0, \quad u^{\prime}(x) \geq 0, \quad u^{\prime \prime}(x) \geq 0, \quad u^{\prime \prime \prime}(x) \leq 0 .
$$

Proof. Conditions (16) follow from Eqs. (11), (13)-(15), respectively, being

$[\sin k t+(1-\cos k t) \tan k] \sin k x+\cos k x$

$$
\geq[\sin k x+(1-\cos k x) \tan k x] \sin k x+\cos k x=\frac{1}{\cos k x} \geq 1,
$$

$$
\begin{aligned}
& {[\sin k t+(1-\cos k t) \tan k] \cos k x-\sin k x } \\
& \geq[\sin k t+(1-\cos k t) \tan k t] \cos k x-\sin k x=(\tan k t-\tan k x) \cos k x \geq 0,
\end{aligned}
$$

for $t \in[x, 1]$.

Therefore, the function $u$ is positive, increasing and convex for compressive axial load. 
We prove here some bounds for the solution of the BVP (1) and (11) for $0 \leq u(x) \leq 1$.

Let us start by providing an upper bound for the solution $u(x)$ to the problem (1) and (4).

Lemma 3.1. Let $u(x)$ be the solution to the BVP (1) and (4), then

$$
u(x) \leq u(1) b(x), \quad \text { for } x \in[0,1],
$$

where

$$
b(x)=\frac{1}{3}\left[6 x^{2}-4 x^{3}+x^{4}-\frac{4 k^{2}}{18-k^{2}}\left(3 x^{2}-5 x^{3}+2 x^{4}\right)\right] .
$$

Proof. Let us introduce the function

$$
h(x)=u(1)\left(2 x^{2}-\frac{4}{3} x 3+\frac{1}{3} x^{4}\right)-\frac{k^{2}}{3} u^{\prime}(1)\left(\frac{1}{2} x^{2}-\frac{5}{6} x^{3}+\frac{1}{3} x^{4}\right)-u(x),
$$

whose derivatives are

$$
\begin{aligned}
& h^{\prime}(x)=4 u(1)\left(x-x^{2}+\frac{1}{3} x^{3}\right)-\frac{k^{2}}{3} u^{\prime}(1)\left(x-\frac{5}{2} x^{2}+\frac{4}{3} x^{3}\right)-u^{\prime}(x), \\
& h^{\prime \prime}(x)=4 u(1)\left(1-2 x+x^{2}\right)-\frac{k^{2}}{3} u^{\prime}(1)\left(1-5 x+4 x^{2}\right)-u^{\prime \prime}(x), \\
& h^{\prime \prime \prime}(x)=-8 u(1)(1-x)+\frac{k^{2}}{3} u^{\prime}(1)(5-8 x)-u^{\prime \prime \prime}(x), \\
& h^{\mathrm{IV}}(x)=8\left[u(1)-\frac{k^{2}}{3} u^{\prime}(1)\right]-u^{\mathrm{IV}}(x), \\
& h^{\mathrm{V}}(x)=-u^{\mathrm{V}}(x)=-\frac{d f(u)}{d u} u^{\prime}(x)+k^{2} u^{\prime \prime \prime}(x) \leq 0 .
\end{aligned}
$$

The latter inequality follows from (16), (1) and (2) for $u(x) \leq 1$, being

$$
\frac{d f(u)}{d u}=\frac{\gamma \beta}{(1-u)^{2}}+\frac{2 \beta}{(1-u)^{3}}+\frac{3 \alpha_{W}}{(1-u)^{4}}+\frac{4 \alpha_{C}}{(1-u)^{5}} \geq 0 .
$$

Therefore, the function $h(x)$ satisfies the following conditions

$$
h(0)=0, h(1)=0, \quad h^{\prime}(0)=0, h^{\prime \prime}(1)=0, h^{\prime \prime \prime}(1)=0 .
$$

By using the mean value theorem, continuity of the function $h(x)$ and conditions $(24)_{1,2}$ imply that there exists $x_{1} \in[0$, 1] such that $h^{\prime}\left(x_{1}\right)=0$. Then, by using conditions $(24)_{3,4}$ there exists $x_{2} \in\left[0, x_{1}\right]$ such that $h^{\prime \prime}\left(x_{2}\right)=0$ and also $x_{3} \in\left[x_{2}, 1\right]$ such that $h^{\prime \prime \prime}\left(x_{3}\right)=0$. Moreover, $h^{\prime \prime \prime}(x)$ is concave being $h^{\mathrm{V}}(x) \leq 0$. It follows that $h^{\prime \prime}(x) \leq 0$ for $x \in\left[x_{2}, 1\right]$ and $h^{\prime \prime}(x) \geq 0$ for $x$ $\in\left[0, x_{2}\right]$, and thus $h^{\prime}(x) \geq 0$ for $x \in\left[0, x_{1}\right]$ and $h^{\prime}(x) \leq 0$ for $x \in\left[x_{1}, 1\right]$. Since $h(0)=h(1)=0$, then it necessarily follows that $h(x) \geq 0$ for $x \in[0,1]$, namely

$$
u(x) \leq u(1)\left(2 x^{2}-\frac{4}{3} x^{3}+\frac{1}{3} x^{4}\right)-\frac{k^{2}}{3} u^{\prime}(1)\left(\frac{1}{2} x^{2}-\frac{5}{6} x^{3}+\frac{1}{3} x^{4}\right), \quad \text { for } x \in[0,1]
$$

Moreover, from the conditions $h^{\prime}(1) \leq 0$ and $h^{\prime \prime}(0) \geq 0$, by using (22) one obtains

$$
h^{\prime}(1)=\frac{4}{3} u(1)-\left(1-\frac{k^{2}}{18}\right) u^{\prime}(1) \leq 0, \quad h^{\prime \prime}(0)=4 u(1)-\frac{k^{2}}{3} u^{\prime}(1)-u^{\prime \prime}(0) \geq 0,
$$

and thus

$$
u^{\prime}(1) \geq \frac{24}{18-k^{2}} u(1), \quad u^{\prime \prime}(0) \leq 4 u(1)-\frac{k^{2}}{3} u^{\prime}(1) \leq 12 \frac{6-k^{2}}{18-k^{2}} u(1) .
$$

The introduction of $(27)_{1}$ in the inequality (25), considering that the term multiplying $u^{\prime}(1)$ is non negative for $x \in[0$, 1], then yields the upper bound (19).

From conditions $(16)_{4}$ and $(27)_{2}$ it follows that

$$
u^{\prime \prime}(x) \leq u^{\prime \prime}(0) \leq 12 \frac{6-k^{2}}{18-k^{2}} u(1)
$$

and thus from (1) and (28) one obtains the following lower bound for $u^{\mathrm{IV}}$

$$
u^{I V}(x) \geq \beta^{*}-12 k^{2} \frac{6-k^{2}}{18-k^{2}} u(1),
$$


being $f(u) \geq \beta^{*}$, where

$$
\beta^{*}=\beta(1+\gamma)+\alpha_{W}+\alpha_{C} .
$$

The result (19) holds also in the absence of the compressive axial load, namely for $k=0$, as stated in the next corollary that follows from Lemma 3.1.

Corollary 3.1. Let $u_{0}(x)$ be the solution to the BVP (1) and (4) for $k=0$ then

$$
u_{0}(x) \leq u(1)\left(2 x^{2}-\frac{4}{3} x^{3}+\frac{1}{3} x^{4}\right) .
$$

The same result was obtained in [35] and it has been recovered recently in [21] as a special case for an elastically constrained cantilever under electrostatic actuation.

In the following we derive a lower bound for the solution $u(x)$ to the BVP (1) and (4).

Lemma 3.2. Let $u(x)$ be the solution to the BVP (1) and (4), then

$$
u(x) \geq u(1) a_{1}(x)+\beta^{*} a_{2}(x) \quad \text { for } x \in[0,1],
$$

where

$$
a_{1}(x)=\frac{1}{2}\left(3 x^{2}-x^{3}\right)-\frac{k^{2}\left(6-k^{2}\right)}{4\left(18-k^{2}\right)}\left(3 x^{2}-5 x^{3}+2 x^{4}\right), \quad a_{2}(x)=\frac{1}{48}\left(3 x^{2}-5 x^{3}+2 x^{4}\right),
$$

Proof. Let us introduce the following function

$$
g(x)=u(x)-\frac{u(1)}{2}-\left(3 x^{2}-x^{3}\right)-\left[\frac{\beta^{*}}{48}-\frac{k^{2}\left(6-k^{2}\right)}{4\left(18-k^{2}\right)} u(1)\right]\left(3 x^{2}-5 x^{3}+2 x^{4}\right),
$$

whose derivatives are

$$
\begin{aligned}
& g^{\prime}(x)=u^{\prime}(x)-\frac{3}{2} u(1)\left(2 x-x^{2}\right)-\left[\frac{\beta^{*}}{48}-\frac{k^{2}\left(6-k^{2}\right)}{4\left(18-k^{2}\right)} u(1)\right]\left(6 x-15 x^{2}+8 x^{3}\right), \\
& g^{\prime \prime}(x)=u^{\prime \prime}(x)-3 u(1)(1-x)-\left[\frac{\beta^{*}}{8}-\frac{3 k^{2}\left(6-k^{2}\right)}{2\left(18-k^{2}\right)} u(1)\right]\left(1-5 x+4 x^{2}\right), \\
& g^{\prime \prime \prime}(x)=u^{\prime \prime \prime}(x)+3 u(1)+\left[\frac{\beta^{*}}{8}-\frac{3 k^{2}\left(6-k^{2}\right)}{2\left(18-k^{2}\right)} u(1)\right](5-8 x), \\
& g^{\mathrm{IV}}(x)=u^{\mathrm{IV}}(x)-\beta^{*}+12 k^{2} \frac{6-k^{2}}{18-k^{2}} u(1) \geq 0,
\end{aligned}
$$

where the latter inequality follows from relation (29). Therefore, the function $g(x)$ satisfies the following conditions

$$
g(0)=0, g(1)=0, g^{\prime}(0)=0, g^{\prime \prime}(1)=0 .
$$

By using the mean value theorem, continuity of $g(x)$ together with conditions $(36)_{1,2}$ imply that there exists $x_{1} \in[0,1]$ such that $g^{\prime}\left(x_{1}\right)=0$. Moreover, by using conditions (36) 3,4 there exists $x_{2} \in\left[0, x_{1}\right]$ such that $g^{\prime \prime}\left(x_{2}\right)=0$ and also $x_{3} \in\left[x_{2}, 1\right]$ such that $g^{\prime \prime \prime}\left(x_{3}\right)=0$. Condition (35) $)_{4}$ implies that $g^{\prime \prime}(x)$ is convex. It follows that $g^{\prime \prime}(x) \leq 0$ for $x \in\left[x_{2}, 1\right]$ and $g^{\prime \prime}(x) \geq 0$ for $x$ $\in\left[0, x_{2}\right]$, and thus $g^{\prime}(x) \geq 0$ for $x \in\left[0, x_{1}\right]$ and $g^{\prime}(x) \leq 0$ for $x \in\left[x_{1}, 1\right]$. Since $g(0)=g(1)=0$, then it necessarily follows that $g(x) \geq 0$ for $x \in[0,1]$, namely

$$
u(x) \geq \frac{u(1)}{2}\left(3 x^{2}-x^{3}\right)+\left[\frac{\beta^{*}}{48}-\frac{k^{2}\left(6-k^{2}\right)}{4\left(18-k^{2}\right)} u(1)\right]\left(3 x^{2}-5 x^{3}+2 x^{4}\right), \quad \text { for } x \in[0,1]
$$

so that the lower bound (32) is attained.

The result (32) holds also in the absence of the compressive axial load, namely for $k=0$, as stated in the next corollary, which follows straight from Lemma 3.2.

Corollary 3.2. Let $u_{0}(x)$ be the solution to the BVP (1) and (4) for $k=0$, then

$$
u_{0}(x) \geq \frac{u(1)}{2}\left(3 x^{2}-x^{3}\right)+\frac{\beta *}{48}\left(3 x^{2}-5 x^{3}+2 x^{4}\right) .
$$

\section{Lower and upper bounds on the pull-in parameters}

In the following we denote with $r=u(1)$ the normalized deflection of the cantilever tip. By using (12) and the estimates (19), (32) and (27) $)_{1}$ on the solution of the BVP (1) and (4), the following lower and upper bounds can be derived for the pull-in parameters $\beta_{P I}$ and $r_{P I}$. 


\subsection{Lower bounds on the pull-in parameters}

By using (19) and (20), from (12) it follows

$$
\beta \geq \frac{r k^{3}-F(r)}{L(r)},
$$

where the following functions can be calculated numerically

$$
\begin{aligned}
& L(r)=\int_{0}^{1} \frac{\sin k t-k t+(1-\cos k t) \tan k}{1-r b(t)}\left(\gamma+\frac{1}{1-r b(t)}\right) d t, \\
& F(r)=\int_{0}^{1} \frac{\sin k t-k t+(1-\cos k t) \tan k}{[1-r b(t)]^{3}}\left(\alpha_{W}+\frac{\alpha_{C}}{1-r b(t)}\right) d t .
\end{aligned}
$$

Inequality (39) defines a lower bound to the exact relation between the normalized voltage $\beta$ and the normalized tip deflection $r$ provided by the solution of the BVP (1) and (4). Therefore, the maximum value of the left hand side of (39) yields the lower bounds $\beta_{l}$ and $r_{l}$ for the pull-in parameters, such that $\beta_{P I} \geq \beta_{l}$ and $r_{P I} \geq r_{l}$. Therefore, the lower bounds for the pull-in parameters are given by the following equations obtained from the stationary condition of the left hand side of (39)

$$
\beta_{l} L\left(r_{l}\right)+F\left(r_{l}\right)=r_{l} k^{3}, \quad \beta_{l} L^{\prime}\left(r_{l}\right)+F^{\prime}\left(r_{l}\right)=k^{3},
$$

where also the following functions can be calculated numerically

$$
\begin{aligned}
& L^{\prime}(r)=\int_{0}^{1} \frac{\sin k t-k t+(1-\cos k t) \tan k}{[1-r b(t)]^{2}}\left(\gamma+\frac{2}{1-r b(t)}\right) b(t) d t, \\
& F^{\prime}(r)=\int_{0}^{1} \frac{\sin k t-k t+(1-\cos k t) \tan k}{[1-r b(t)]^{4}}\left(3 \alpha_{W}+\frac{4 \alpha_{C}}{1-r b(t)}\right) b(t) d t .
\end{aligned}
$$

\subsection{Upper bounds on the pull-in parameters}

By using (32) and (33), from (12) it follows

$$
r \geq[\beta K(r, \beta)+H(r, \beta)] / k^{3},
$$

where

$$
\begin{aligned}
& K(r, \beta)=\int_{0}^{1} \frac{\sin k t-k t+(1-\cos k t) \tan k}{1-r a_{1}(t)-\beta^{*} a_{2}(t)}\left(\gamma+\frac{1}{1-r a_{1}(t)-\beta^{*} a_{2}(t)}\right) d t, \\
& H(r, \beta)=\int_{0}^{1} \frac{\sin k t-k t+(1-\cos k t) \tan k}{\left[1-r a_{1}(t)-\beta^{*} a_{2}(t)\right]^{3}}\left(\alpha_{W}+\frac{\alpha_{C}}{1-r a_{1}(t)-\beta^{*} a_{2}(t)}\right) d t,
\end{aligned}
$$

where the parameter $\beta^{*}$ has been defined in (30).

The inequality (43) defines an upper bound to the exact relation between the parameters $\beta$ and $r$. Indeed, by equating both sides of condition (43) we obtain an implicit relation for $\beta$ as a function of $r$ that is greater than the exact relation provided by the solution of the BVP (1) and (4). The maximum value of the implicit function $\beta(r)$ obtained from (43) by using the stationary condition

$$
\frac{d \beta}{d r}=0
$$

then yields the upper bounds $\beta_{u}$ and $r_{u}$ for the pull-in parameters, such that $\beta_{P I} \leq \beta_{u}$ and $r_{P I} \leq r_{u}$. Therefore, the upper bounds for the pull-in parameters are given by the following conditions

$$
\beta_{u} K\left(r_{u}, \beta_{u}\right)+H\left(r_{u}, \beta_{u}\right)=k^{3} r_{u}, \quad \beta_{u} K^{\prime}\left(r_{u}, \beta_{u}\right)+H^{\prime}\left(r_{u}, \beta_{u}\right)=k^{3},
$$

where

$$
\begin{aligned}
& K^{\prime}(r, \beta)=\int_{0}^{1} \frac{\sin k t-k t+(1-\cos k t) \tan k}{\left[1-r a_{1}(t)-\beta^{*} a_{2}(t)\right]^{2}}\left(\gamma+\frac{2}{1-r a_{1}(t)-\beta^{*} a_{2}(t)}\right) a_{1}(t) d t, \\
& H^{\prime}(r, \beta)=\int_{0}^{1} \frac{\sin k t-k t+(1-\cos k t) \tan k}{\left[1-r a_{1}(t)-\beta^{*} a_{2}(t)\right]^{4}}\left(3 \alpha_{W}+\frac{4 \alpha_{C}}{1-r a_{1}(t)-\beta^{*} a_{2}(t)}\right) a_{1}(t) d t .
\end{aligned}
$$


Table 1

Lower and upper bounds for the pull-in parameters of a micro/nanocantilever subjected to a compressive axial load with $k=0.5$, for several values of the vdW and Casimir parameters $\alpha_{W}, \alpha_{C}$, and geometric ratio $d / w$.

\begin{tabular}{|c|c|c|c|c|c|c|c|c|c|c|c|c|c|}
\hline \multicolumn{2}{|c|}{$k=0.5$} & \multicolumn{4}{|l|}{$d / w=0$} & \multicolumn{4}{|l|}{$d / w=1$} & \multicolumn{4}{|l|}{$d / w=2$} \\
\hline$\alpha_{W}$ & $\overline{\alpha_{C}}$ & $\overline{r_{l}}$ & $\beta_{l}$ & $r_{u}$ & $\beta_{u}$ & $\overline{r_{l}}$ & $\beta_{l}$ & $r_{u}$ & $\beta_{u}$ & $\overline{r_{l}}$ & $\beta_{l}$ & $r_{u}$ & $\beta_{u}$ \\
\hline 0.0 & 0.0 & 0.4433 & 1.5112 & 0.4520 & 1.5374 & 0.4873 & 1.0564 & 0.4960 & 1.0736 & 0.5139 & 0.8181 & 0.5226 & 0.8309 \\
\hline 0.0 & 0.2 & 0.3771 & 1.0939 & 0.3853 & 1.1194 & 0.3978 & 0.7437 & 0.4060 & 0.7602 & 0.4086 & 0.5650 & 0.4168 & 0.5772 \\
\hline 0.0 & 0.4 & 0.3347 & 0.7260 & 0.3428 & 0.7513 & 0.3461 & 0.4858 & 0.3543 & 0.5022 & 0.3518 & 0.3655 & 0.3601 & 0.3777 \\
\hline 0.0 & 0.6 & 0.3022 & 0.3869 & 0.3103 & 0.4121 & 0.3075 & 0.2559 & 0.3159 & 0.2723 & 0.3102 & 0.1913 & 0.3186 & 0.2035 \\
\hline 0.0 & 0.8 & 0.2753 & 0.0679 & 0.2835 & 0.0930 & 0.2762 & 0.0445 & 0.2846 & 0.0609 & 0.2766 & 0.0331 & 0.2852 & 0.0453 \\
\hline 0.0 & 1.0 & 0.2522 & -0.236 & 0.2604 & -0.211 & 0.2494 & -0.153 & 0.2580 & -0.137 & 0.2481 & -0.114 & 0.2568 & -0.101 \\
\hline 0.0 & 0.0 & 0.4433 & 1.5112 & 0.4520 & 1.5374 & 0.4873 & 1.0564 & 0.4960 & 1.0736 & 0.5139 & 0.8181 & 0.5226 & 0.8309 \\
\hline 0.2 & 0.0 & 0.4168 & 1.2164 & 0.4255 & 1.2426 & 0.4468 & 0.8400 & 0.4556 & 0.8572 & 0.4635 & 0.6445 & 0.4723 & 0.6573 \\
\hline 0.4 & 0.0 & 0.3945 & 0.9303 & 0.4032 & 0.9567 & 0.4150 & 0.6363 & 0.4238 & 0.6537 & 0.4259 & 0.4849 & 0.4348 & 0.4979 \\
\hline 0.6 & 0.0 & 0.3751 & 0.6513 & 0.3838 & 0.6777 & 0.3882 & 0.4419 & 0.3971 & 0.4594 & 0.3950 & 0.3350 & 0.4041 & 0.3481 \\
\hline 0.8 & 0.0 & 0.3577 & 0.3781 & 0.3664 & 0.4046 & 0.3648 & 0.2547 & 0.3738 & 0.2724 & 0.3684 & 0.1923 & 0.3776 & 0.2055 \\
\hline 1.0 & 0.0 & 0.3419 & 0.1098 & 0.3507 & 0.1364 & 0.3438 & 0.0735 & 0.3530 & 0.0913 & 0.3448 & 0.0553 & 0.3542 & 0.0686 \\
\hline
\end{tabular}

Table 2

Lower and upper bounds for the pull-in parameters of a micro/nanocantilever subjected to a compressive axial load with $k=1$, for several values of the vdW and Casimir parameters $\alpha_{W}, \alpha_{C}$, and geometric ratio $d / w$.

\begin{tabular}{|c|c|c|c|c|c|c|c|c|c|c|c|c|c|}
\hline \multicolumn{2}{|c|}{$k=1$} & \multicolumn{4}{|l|}{$d / w=0$} & \multicolumn{4}{|l|}{$d / w=1$} & \multicolumn{4}{|l|}{$d / w=2$} \\
\hline$\overline{\alpha_{W}}$ & $\overline{\alpha_{C}}$ & $\overline{r_{l}}$ & $\beta_{l}$ & $r_{u}$ & $\beta_{u}$ & $\overline{r_{l}}$ & $\beta_{l}$ & $r_{u}$ & $\beta_{u}$ & $\overline{r_{l}}$ & $\beta_{l}$ & $r_{u}$ & $\beta_{u}$ \\
\hline 0.0 & 0.0 & 0.4460 & 1.0217 & 0.4545 & 1.0424 & 0.4901 & 0.7141 & 0.4987 & 0.7278 & 0.5167 & 0.5529 & 0.5255 & 0.5632 \\
\hline 0.0 & 0.2 & 0.3573 & 0.6174 & 0.3654 & 0.6375 & 0.3728 & 0.4161 & 0.3812 & 0.4293 & 0.3808 & 0.3145 & 0.3892 & 0.3243 \\
\hline 0.0 & 0.4 & 0.3054 & 0.2711 & 0.3135 & 0.2907 & 0.3109 & 0.1793 & 0.3194 & 0.1922 & 0.3137 & 0.1341 & 0.3223 & 0.1437 \\
\hline 0.0 & 0.6 & 0.2666 & -0.045 & 0.2748 & -0.025 & 0.2658 & -0.029 & 0.2744 & -0.017 & 0.2654 & -0.022 & 0.2742 & -0.012 \\
\hline 0.0 & 0.8 & 0.2351 & -0.340 & 0.2433 & -0.321 & 0.2294 & -0.220 & 0.2381 & -0.207 & 0.2267 & -0.162 & 0.2356 & -0.153 \\
\hline 0.0 & 1.0 & 0.2082 & -0.620 & 0.2165 & -0.601 & 0.1986 & -0.397 & 0.2073 & -0.385 & 0.1940 & -0.292 & 0.2030 & -0.283 \\
\hline 0.0 & 0.0 & 0.4460 & 1.0217 & 0.4545 & 1.0424 & 0.4901 & 0.7141 & 0.4987 & 0.7278 & 0.5167 & 0.5529 & 0.5255 & 0.5632 \\
\hline 0.2 & 0.0 & 0.4082 & 0.7291 & 0.4168 & 0.7499 & 0.4333 & 0.5009 & 0.4421 & 0.5148 & 0.4470 & 0.3830 & 0.4559 & 0.3933 \\
\hline 0.4 & 0.0 & 0.3782 & 0.4482 & 0.3868 & 0.4690 & 0.3916 & 0.3041 & 0.4006 & 0.3180 & 0.3986 & 0.2306 & 0.4078 & 0.2410 \\
\hline 0.6 & 0.0 & 0.3528 & 0.1761 & 0.3616 & 0.1969 & 0.3576 & 0.1183 & 0.3668 & 0.1323 & 0.3600 & 0.0891 & 0.3694 & 0.0995 \\
\hline 0.8 & 0.0 & 0.3307 & -0.089 & 0.3395 & -0.068 & 0.3284 & -0.059 & 0.3378 & -0.046 & 0.3273 & -0.044 & 0.3370 & -0.034 \\
\hline 1.0 & 0.0 & 0.3108 & -0.349 & 0.3197 & -0.328 & 0.3027 & -0.230 & 0.3122 & -0.217 & 0.2987 & -0.172 & 0.3085 & -0.162 \\
\hline
\end{tabular}

Table 3

Lower and upper bounds for the pull-in parameters of a micro/nanocantilever subjected to a compressive axial load with $k=1.2$, for several values of the vdW and Casimir parameters $\alpha_{W}, \alpha_{C}$, and geometric ratio $d / w$.

\begin{tabular}{|c|c|c|c|c|c|c|c|c|c|c|c|c|c|}
\hline \multicolumn{2}{|c|}{$k=1.2$} & \multicolumn{4}{|l|}{$d / w=0$} & \multicolumn{4}{|l|}{$d / w=1$} & \multicolumn{4}{|l|}{$d / w=2$} \\
\hline$\alpha_{W}$ & $\alpha_{C}$ & $r_{l}$ & $\beta_{l}$ & $r_{u}$ & $\beta_{u}$ & $r_{l}$ & $\beta_{l}$ & $r_{u}$ & $\beta_{u}$ & $r_{l}$ & $\beta_{l}$ & $r_{u}$ & $\beta_{u}$ \\
\hline 0.0 & 0.0 & 0.4476 & 0.7249 & 0.4556 & 0.7399 & 0.4918 & 0.5066 & 0.4999 & 0.5166 & 0.5185 & 0.3922 & 0.5267 & 0.3998 \\
\hline 0.0 & 0.2 & 0.3350 & 0.3342 & 0.3427 & 0.3485 & 0.3458 & 0.2233 & 0.3538 & 0.2327 & 0.3512 & 0.1679 & 0.3593 & 0.1749 \\
\hline 0.0 & 0.4 & 0.2741 & 0.0079 & 0.2819 & 0.0217 & 0.2743 & 0.0052 & 0.2825 & 0.0142 & 0.2744 & 0.0038 & 0.2827 & 0.0106 \\
\hline 0.0 & 0.6 & 0.2296 & -0.287 & 0.2374 & -0.274 & 0.2230 & -0.185 & 0.2312 & -0.176 & 0.2199 & -0.136 & 0.2283 & -0.130 \\
\hline 0.0 & 0.8 & 0.1937 & -0.561 & 0.2016 & -0.549 & 0.1819 & -0.359 & 0.1902 & -0.350 & 0.1764 & -0.262 & 0.1849 & -0.257 \\
\hline 0.0 & 1.0 & 0.1633 & -0.821 & 0.1712 & -0.809 & 0.1472 & -0.518 & 0.1555 & -0.511 & 0.1397 & -0.378 & 0.1483 & -0.373 \\
\hline 0.0 & 0.0 & 0.4476 & 0.7249 & 0.4556 & 0.7399 & 0.4918 & 0.5066 & 0.4999 & 0.5166 & 0.5185 & 0.3922 & 0.5267 & 0.3998 \\
\hline 0.2 & 0.0 & 0.3967 & 0.4349 & 0.4048 & 0.4499 & 0.4165 & 0.2971 & 0.4249 & 0.3071 & 0.4271 & 0.2263 & 0.4357 & 0.2338 \\
\hline 0.4 & 0.0 & 0.3586 & 0.1597 & 0.3668 & 0.1746 & 0.3647 & 0.1074 & 0.3734 & 0.1174 & 0.3679 & 0.0810 & 0.3768 & 0.0885 \\
\hline 0.6 & 0.0 & 0.3273 & -0.105 & 0.3356 & -0.091 & 0.3236 & -0.070 & 0.3325 & -0.060 & 0.3218 & -0.052 & 0.3310 & -0.045 \\
\hline 0.8 & 0.0 & 0.3004 & -0.363 & 0.3088 & -0.348 & 0.2889 & -0.239 & 0.2980 & -0.229 & 0.2833 & -0.178 & 0.2927 & -0.170 \\
\hline 1.0 & 0.0 & 0.2766 & -0.613 & 0.2850 & -0.599 & 0.2585 & -0.400 & 0.2677 & -0.391 & 0.2499 & -0.297 & 0.2594 & -0.290 \\
\hline
\end{tabular}

\section{Results}

The lower and upper bounds predicted by the present analytical approach for the normalized pull-in voltage $\beta_{l}$ and $\beta_{u}$ and for the normalized pull-in deflection $r_{u}$ and $r_{l}$ have been reported in Tables 1-3 for some particular set of the 188 parameters $d / w, \alpha_{W}$ and $\alpha_{C}$ and for specific values of the axial load parameter $k$ defined in (5) ranging between 0 and 1.5 . 189 It can be observed that the results for vanishing compressive axial load, namely for $k=0$, recover those found in [21], thus 190 validating the present approach. Moreover, if the surface intermolecular parameters are greater than their critical values 

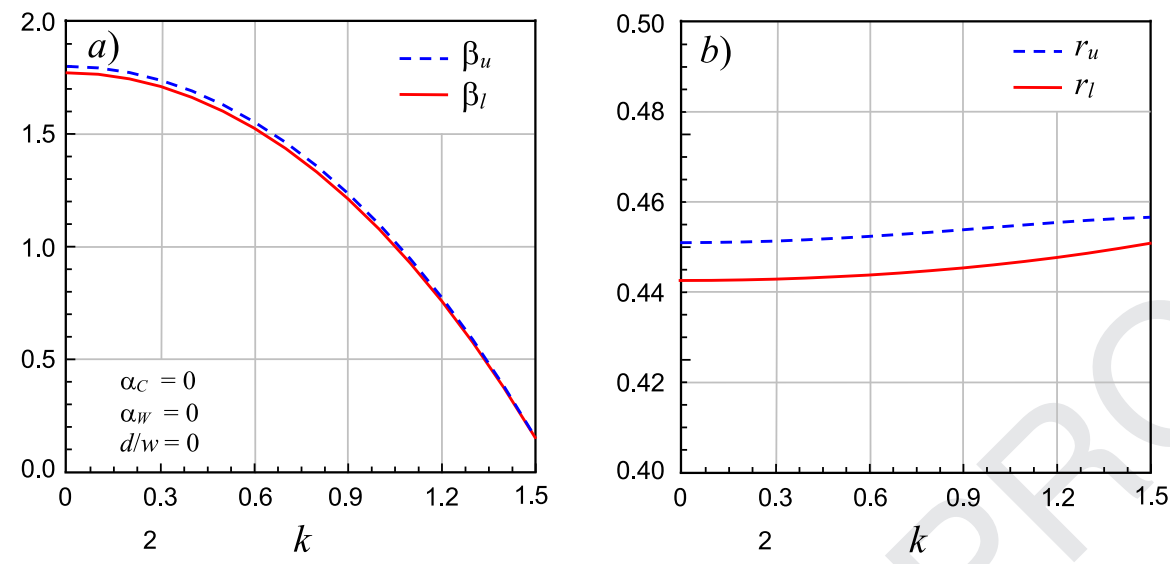

Fig. 2. Variations of lower and upper bounds for the pull-in voltage $\beta_{l}$ and $\beta_{u}$ (a) and tip deflection $r_{l}$ and $r_{u}$ (b) with the axial load parameter $k$ for a microcantilever, for negligible fringing fields and intermolecular surface forces.

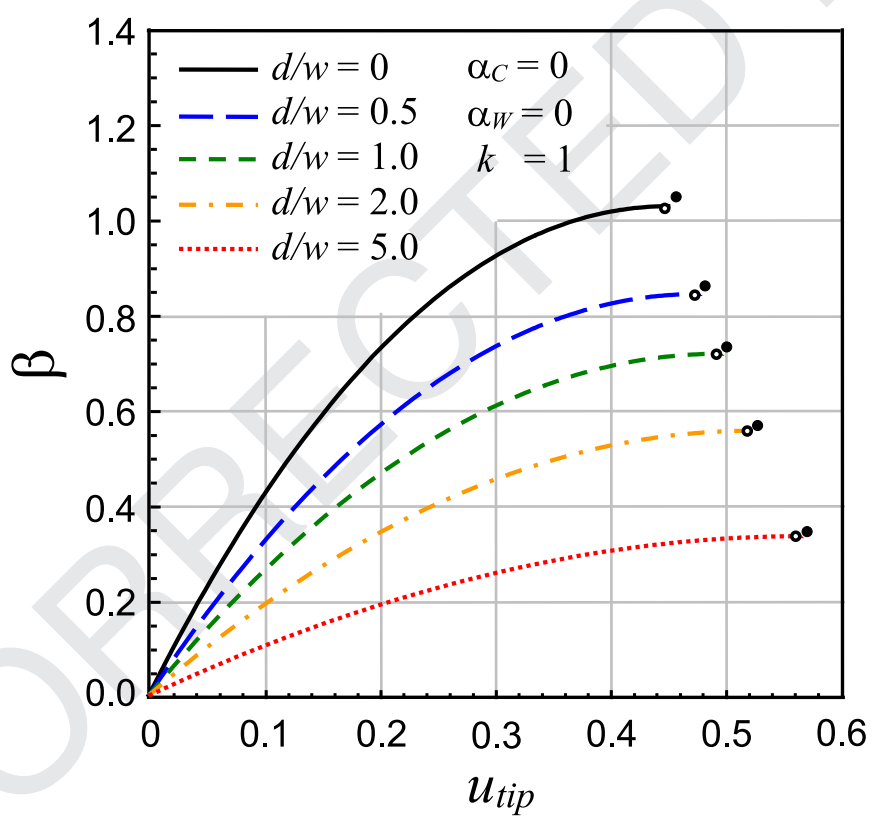

Fig. 3. Numerical results for the tip displacement $u_{\text {tip }}$ as a function of the electrostatic loading parameters $\beta$ provided by the shooting method, for $k=1$ and for some values of the geometric ratio $d / w$. The lower and upper bounds of the pull-in parameters provided by the present analytical approach are denoted by small circles and small points, respectively.

that cause pull-in instability in the absence of electrostatic actuation and axial load, then a repulsive electrostatic force characterized by negative values of $\beta$ is required to prevent pull-in instability and the occurring of stiction. The results obtained for $\alpha_{W}=\alpha_{C}=0$, namely by neglecting the contribution of Casimir or vdW attractions, are significant for MEMS, which are not affected by these forces.

The effects of the axial load parameter $k$ on the lower and upper bounds for the pull-in parameters $\beta_{\mathrm{PI}}$ and $r_{\mathrm{PI}}$ are shown in Fig. 2 for vanishing small fringing and intermolecular surface forces $\left(d / w=\alpha_{C}=\alpha_{W}=0\right)$. These results clearly display that the lower and upper analytical bounds are very close each other for $k$ ranging between 0 and $\pi / 2$, and thus they provide very accurate estimates of the actual pull-in parameters $\beta_{P I}$ and $r_{P I}$. Moreover, the pull-in voltage becomes null as the axial load parameter tends to its limit value $k=\pi / 2$, corresponding to the classical elastic buckling load of a EB cantilever beam.

The analytical bounds have been validated by comparison with the numerical solution of the nonlinear BVP (1) and (4) calculated by using the command NDSolve of the Mathematica software (version number 8), which exploits the shooting method for the numerical integration of two point BVP [36]. The variation of the tip deflection of the nanocantilever $r=u(1)$ with the electrostatic loading parameter $\beta$ obtained from the Mathematica software are reported in Fig. 3 for the same value of the axial load parameter, $k=1$, and for different values of the geometric ratio $d / w$. The largest pull-in voltage is attained for $d / w=0$, namely for a vanishing small fringing field occurring when the separation distance $d$ is much smaller then the beam width $w$. If the effects of fringing fields become more relevant, as it occurs for large values of the ratio $d / w$, then the pull-in voltage $\beta_{P I}$ decreases, whereas the pull-in tip deflection $r_{P I}$ increases. In the following figures, we denote with small 

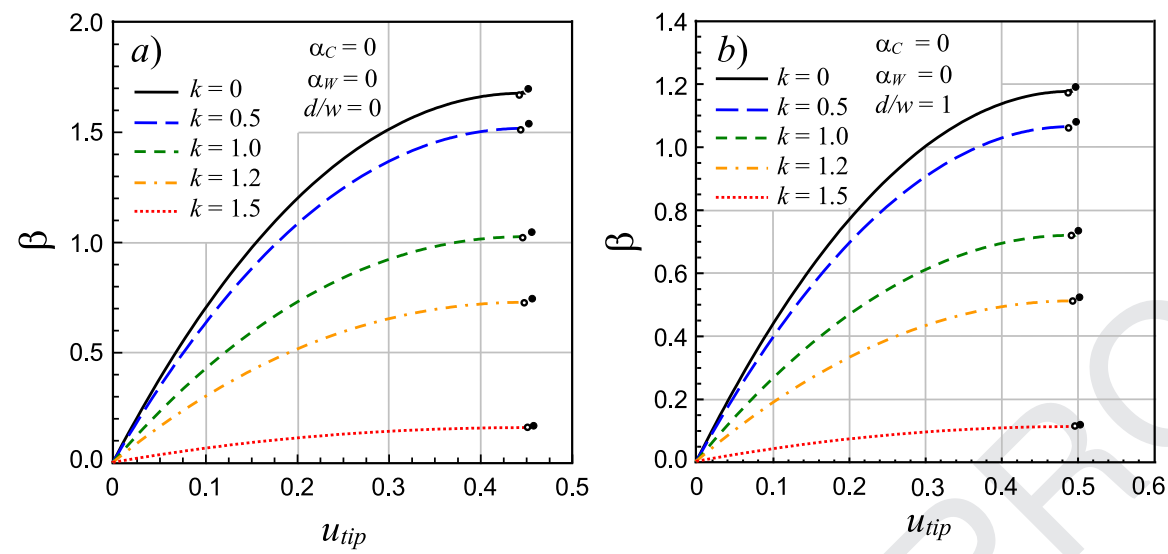

Fig. 4. Numerical results for the tip displacement $u_{\text {tip }}$ as a function of the electrostatic loading parameters $\beta$ provided by the shooting method, for some values $k$ ranging between 0 and 1.5, for negligible intermolecular forces $\left(\alpha_{W}=\alpha_{C}=0\right)$ and for geometric ratios $d / w=0(a)$ and $d / w=1(b)$. The lower and upper bounds of the pull-in parameters provided by the present analytical approach are denoted by small circles and small points, respectively.
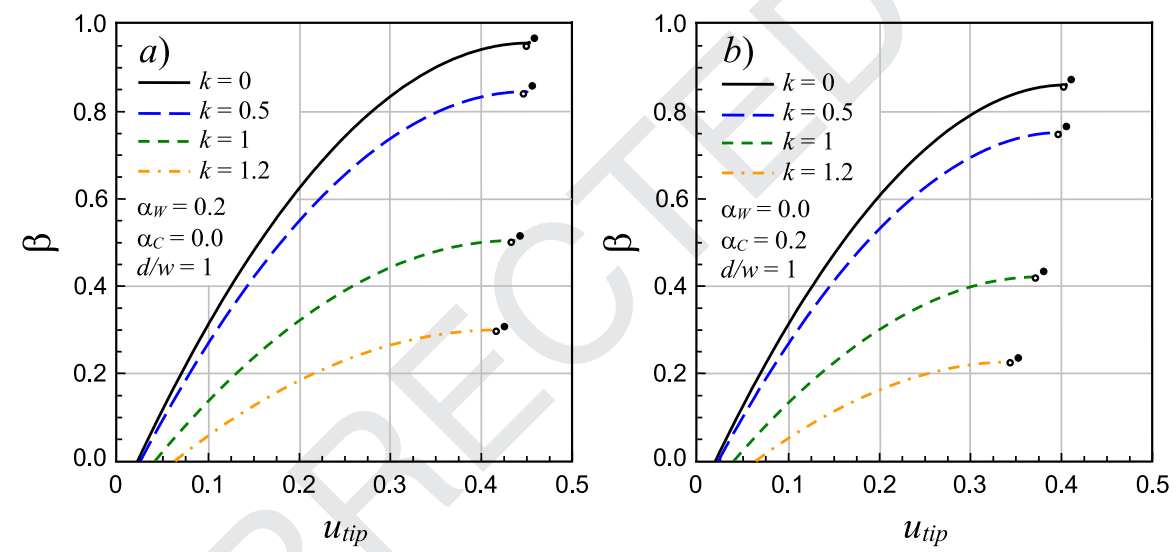

Fig. 5. Numerical results for the tip displacement $u_{\text {tip }}$ as a function of the electrostatic loading parameters $\beta$ provided by the shooting method, for some values $k$ ranging between 0 and 1.5, for the geometric ratios $d / w=1$, for vdW parameters $\alpha_{W}=0.2$ (a) and for Casimir parameters $\alpha_{C}=0.2$ (b). The lower and upper bounds of the pull-in parameters provided by the present analytical approach are denoted by small circles and small points, respectively.

circles and small points the lower and upper bounds for the pull-in parameters, respectively. It can be observed that the exact pull-in parameters, namely the maximum point attained by the numerical curves plotted in Fig. 3, fall between the lower and upper bounds.

The variations of the deflection $r$ of the cantilever tip with the electrostatic loading parameter $\beta$ obtained from the Mathematica software are plotted in Figs. 4 and 5 for some specific set of the parameters $d / w, \alpha_{W}$ and $\alpha_{C}$ and for a range of values of the axial load parameter $k$ between 0 and 1.5. These results show that the combined effects of fringing effect, intermolecular surface forces and compressive axial load significantly reduce the pull-in voltage. In Fig. 4, no noticeable effect of the axial load can be detected on the pull-in deflection, which is almost independent of $k$ in the absence of intermolecular surface forces. A moderate reduction for the pull-in deflection as the axial load parameter $k$ is increased is instead observed in Fig. 5, due to the combined effects of the axial load and intermolecular forces. Note that the lower bounds for $\beta$ and $r$ almost coincide with the exact pull-in parameters predicted by numerical investigation. Moreover, Fig. 5 shows that the intermolecular surface forces cause a deflection of the nanocantilever also in the absence of electric voltage, namely for $\beta=0$.

The variations of the upper and lower bounds $\beta_{u}$ and $\beta_{l}$ with $\alpha_{W}$ and $\alpha_{C}$ are plotted in Fig. 6 for the range of values of the axial load parameter $k$. This plot shows that the compressive axial load and the intermolecular surface forces have similar effects on the electrostatic pull-in parameter, consisting in a significant reduction of the pull-in voltage. It can be observed that the analytical estimates are very close and thus accurate for the full ranges of variation of the axial load parameter and intermolecular surface forces.

The variations of the tip displacement $u_{t i p}$ with the vdW and Casimir parameters, $\alpha_{W}$ and $\alpha_{C}$, provided by the numerical integration procedure implemented in the Mathematica software are presented in Figs. 7a,b for vanishing electric actuation $(\beta=0)$ and for several values of the axial load parameter $k$. These plots reveal that pull-in instability may occur even in the absence of electric voltage if the vdW or Casimir parameters exceed their maximum value $\alpha_{W 0}$ or $\alpha_{C 0}$. Moreover they show that an increase in the compressive axial load reduces the limit values of the intermolecular surface forces, but has 

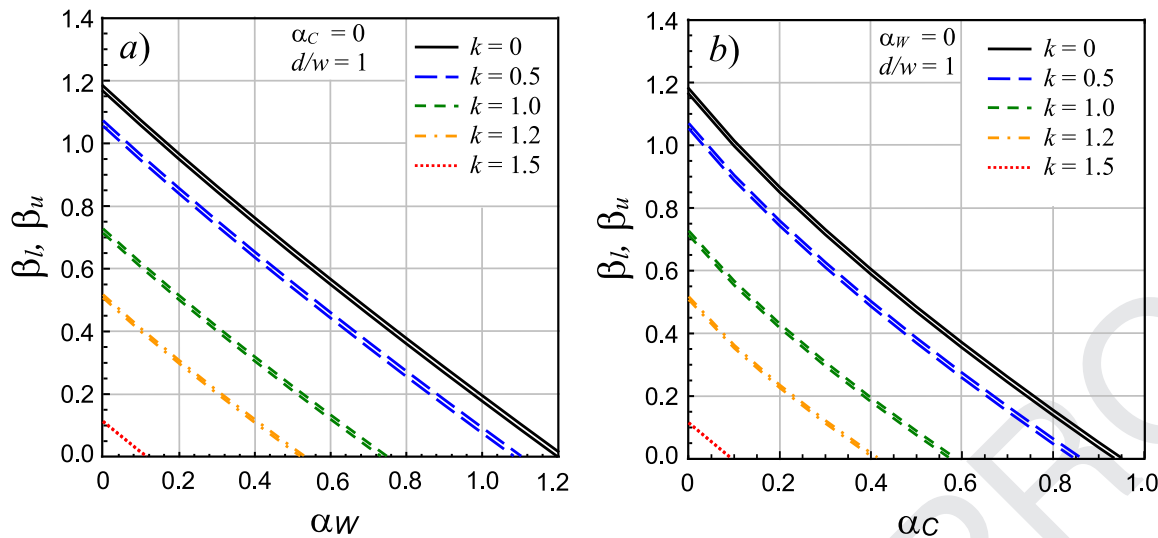

Fig. 6. Effects of vdW (a) and Casimir (b) attractions on the lower and upper analytical bounds for the pull-in voltage, for a range of values of $k$ between 0 and 1.5 .
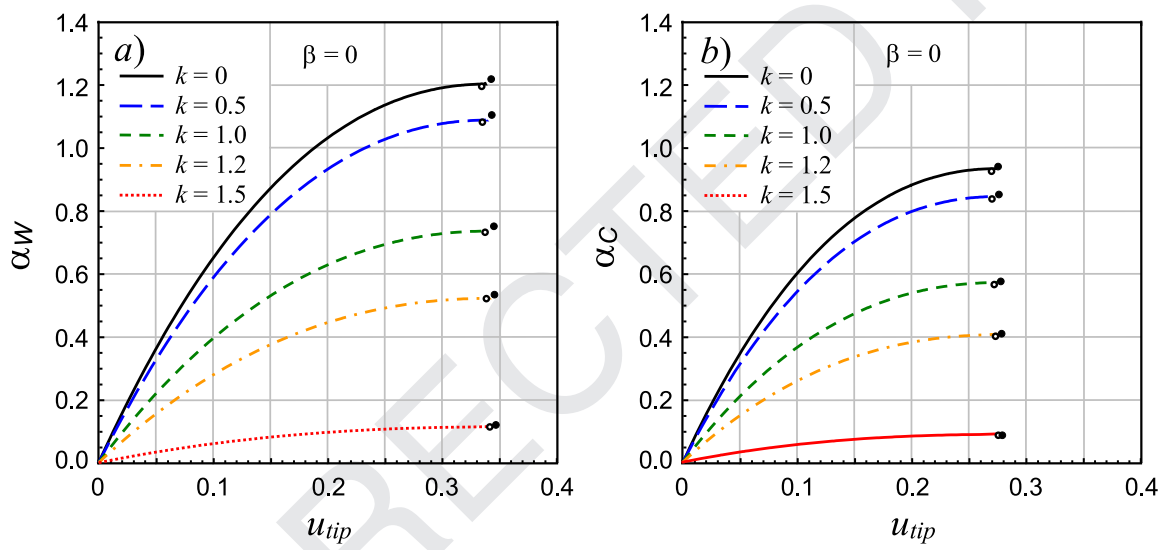

Fig. 7. Numerical results for the tip displacement $u_{\text {tip }}$ as a function of the vdW (a) and Casimir (b) parameters $\alpha_{W}$ and $\alpha_{C}$ in the absence of electrostatic actuation $(\beta=0)$ provided by the shooting method for some values $k$ ranging between 0 and 1.5. The lower and upper bounds of the critical vdW and Casimir parameters provided by the analytical approach are denoted by small circles and small points, respectively.

Table 4

Lower and upper bounds for the critical vdW and Casimir parameters $\alpha_{W}, \alpha_{C}$, and corresponding critical tip deflections $r_{W}$ and $r_{C}$.

\begin{tabular}{lllllllll}
\hline$k$ & $\alpha_{W l}$ & $r_{W l}$ & $\alpha_{W u}$ & $r_{W u}$ & $\alpha_{C l}$ & $r_{C l}$ & $\alpha_{C u}$ & $r_{C u}$ \\
\hline 0.0 & 1.1967 & 0.3350 & 1.2171 & 0.3423 & 0.9326 & 0.2694 & 0.9492 & 0.2756 \\
0.5 & 1.0828 & 0.3357 & 1.1030 & 0.3430 & 0.8439 & 0.2700 & 0.8602 & 0.2762 \\
1.0 & 0.7323 & 0.3379 & 0.7480 & 0.3449 & 0.5708 & 0.2717 & 0.5834 & 0.2777 \\
1.2 & 0.5197 & 0.3392 & 0.5310 & 0.3458 & 0.4051 & 0.2728 & 0.4141 & 0.2784 \\
1.5 & 0.1129 & 0.3418 & 0.1151 & 0.3466 & 0.0881 & 0.2750 & 0.0898 & 0.2790 \\
\hline
\end{tabular}

no significant influence on the pull-in deflection. In these plots, the lower and upper bounds for the parameter $\alpha_{W 0}$ and $\alpha_{C O}$ causing the pull-in of the device and the corresponding pull-in deflection $r_{W 0}$ and $r_{C O}$ are denoted by small circles and points. The analytical predictions for the lower and upper bounds agree very well with the numerical results obtained by the Mathematica software.

Since the Casimir force is effective at larger distances than the vdW force, then pull-in instability caused by the effect of the Casimir force is found to occur at smaller tip deflections, and thus at larger separation distances between the electrodes, than the pull-in tip instability caused by the action of the vdW force. The lower and upper bounds for the critical vdW and Casimir parameters $\alpha_{W_{0}}$ and $\alpha_{C 0}$ for a compressed nanocantilever in the absence of electrostatic actuation can be found also in Table 4 for the considered range of values of the axial load parameter $k$.

\section{Conclusions}

A useful analytical method for accurately predicting the pull-in instability of a micro- or nanocantilever subjected to electrostatic actuation, compressive axial load and vdW or Casimir attractions has been proposed as an alternative to the numerical solution of the extremely nonlinear BVP. After the influence of the compressive axial load on the pull-in 
instability has been examined, the accuracy of the analytical lower and upper bounds has been verified by comparison with the numerical solution of the nonlinear BVP obtained by using the shooting method procedure available in the Mathematica package. In particular, the lower bounds are found to be very close to the exact pull-in parameters.

The provided estimates make the present study particularly significant for developing new practical applications in the field of MEMS and NEMS and make it crucial for the validation of many numerical investigations. We indeed recover that the interaction between the compressive axial load, intermolecular surface forces and fringing field can significantly reduce the pull-in voltage [10-12]. If the contribution of the compressive axial load is neglected then the pull-in voltage may be considerably overestimated and this inaccuracy may lead to unexpected damage during device operation. Therefore, the present investigation may be very helpful for assuring the safe operation of MEMS and NEMS current devices, since it allows avoiding any potential breakage by predicting accurate bounds for the critical pull-in characteristics.

\section{Acknowledgment}

Support from the Italian "Gruppo Nazionale di Fisica Matematica" INdAM-GNFM is gratefully acknowledged.

\section{References}

[1] O.Y. Loh, H.D. Espinosa, Nanoelectromechanical contact switches, Nat. Nanotech. 7 (2012) 283-295.

[2] A.M. Ionescu, Nano-electro-mechanical (NEM) memory devices, in: A.N. Chen, J. Hutchby, V. Zhirnov, G. Bourianoff (Eds.), Emerging Nanoelectronic Devices, John Wiley \& Sons Ltd., 2014, pp. 123-136.

[3] A. Ramezani, A. Alasty, J. Akbari, Closed-form solutions of the pull-in instability in nano-cantilevers under electrostatic and intermolecular surface forces, Int. J. Solids Struct. 44 (2007) 4925-4941.

[4] W.H. Lin, Y.P. Zhao, Pull-in instability of micro-switch actuators, model review, Int. J. Nonlin. Sci. Numer. Simul. 9 (2008) 175-183.

[5] W.C. Chuang, H.L. Lee, P.Z. Chang, Y.C. Hu, Review on the modeling of electrostatic MEMS, Sensors 10 (2010) 6149-6171.

[6] W.M. Zhang, H. Yan, Z.K. Peng, G. Meng, Electrostatic pull-in instability in MEMS/NEMS. A review, Sens. Actuators A Phys 214 (2014) 187-218.

[7] S.P. Timoshenko, J.M. Gere, Theory of Elastic Stability, second ed., Dover, New York, 2009.

[8] D. Elata, S. Abu-Salih, Analysis of a novel method for measuring residual stress in micro-systems, J. Micromech. Microeng. 15 (2005) 921.

[9] S. Abu-Salih, D. Elata, Experimental validation of electromechanical buckling, J. Microelectro-mech. Syst. 15 (2006) 1656-1662.

[10] S.A. Emam, A general nonlocal nonlinear model for buckling of nanobeams, Appl. Math. Model 37 (2013) 6929-6939.

[11] M.A. Eltaher, M.E. Khater, S.A. Emam, A review on nonlocal elastic models for bending, buckling, vibrations, and wave propagation of nanoscale beams, Appl. Math. Model 40 (2016) 4109-4128.

[12] M. Shaat, A. Abdelkefi, Buckling characteristics of nanocrystalline nano-beams, Int. J. Mech. Mater. Des. (2017) 1-19, doi:10.1007/s10999-016-9361-2.

[13] E. Buks, M.L. Roukes, Stiction, adhesion energy, and the Casimir effect in micromechanical systems, Phys. Rev. B 63 (2001) 033402.

[14] S. Basu, A. Prabhakar, E. Bhattacharya, Estimation of stiction force from electrical and optical measurements on cantilever beams, J. Microelectromech. Syst. 16 (2007) 1254-1262.

[15] T.J. Wagner, D. Vella, Switch on, switch off: stiction in nanoelectromechanical switches, Nanotech 24 (2013) 275501.

[16] E.M. Abdel-Rahman, M.I. Younis, A.H. Nayfeh, Characterization of the mechanical behavior of an electrically actuated microbeam, J. Micromech. Microeng. 12 (2002) 759-766.

[17] L.X. Zhang, Y.P. Zhao, Electromechanical model of RF MEMS switches, Microsys. Tech. 9 (2003) 420-426.

[18] Y. Zhang, Y.P. Zhao, Numerical and analytical study on the pull-in instability of micro-structure under electrostatic loading, Sens. Actuators A Phys. 127 (2006) 366-380.

[19] C.W. Lim, C. Li, J.-L. Yu, The effects of stiffness strengthening nonlocal stress and axial tension on free vibration of cantilever nanobeams, Interact. Multiscale Mech. 2 (2009) 223-233.

[20] Y.Y. Zhang, C.M. Wang, N. Challamel, Bending, buckling and vibration of hybrid nonlocal beams, J. Eng. Mech. ASCE 136 (2010) $562-574$

[21] E. Radi, G. Bianchi, L. di Ruvo, Upper and lower bounds for the pull-in parameters of a micro- or nanocantilever on a flexible support, Int. J. Nonlin. Mech. 92 (2017) 176-186.

[22] R. Soroush, A. Koochi, A.S. Kazemi, A. Noghrehabadi, H. Haddadpour, M. Abadyan, Investigating the effect of Casimir and van der Waals attractions on the electrostatic pull-in instability of nano-actuators, Phys. Scr. 82 (2010) 045801.

[23] A.C. Eringen, Nonlocal Continuum Field Theories, Springer, 2002.

[24] H.S. Park, Surface stress effects on the critical buckling strains of silicon nanowires, Comput. Mater. Sci. 51 (2012) 396-401.

[25] S. Cuenot, C. Frétigny, S. Demoustier-Champagne, B. Nysten, Surface tension effect on the mechanical properties of nanomaterials measured by atomic force microscopy, Phys. Rev. B 69 (2004) 165410.

[26] G.-F. Wang, X.-Q. Feng, Surface effects on buckling of nanowires under uniaxial compression, Appl. Phys. Lett. 94 (2009) 141913.

[27] N. Challamel, I. Elishakoff, Surface stress effects may induce softening: Euler-Bernoulli and Timoshenko buckling solutions, Phys. E Low Dimens. Syst. Nanostruct. 44 (2012) 1862-1867.

[28] A. Farrokhabadi, A. Mohebshahedin, R. Rach, J.S. Duan, An improved model for the cantilever NEMS actuator including the surface energy, fringing field and Casimir effects, Phys. E Low Dimens. Syst. Nanostruct. 75 (2016) 202-209.

[29] Y.T. Beni, A. Koochi, M. Abadyan, Theoretical study of the effect of Casimir force, elastic boundary conditions and size dependency on the pull-in instability of beam-type NEMS, Phys. E Lowdim. Sys. Nanostruct. 43 (2011) 979-988.

[30] W.D. Yang, X. Wang, C.Q. Fang, G. Lu, Electromechanical coupling characteristics of carbon nanotube reinforced cantilever nano-actuator, Sens. Actuators A Phys. 220 (2014) 178-187.

[31] A. Koochi, A. Kazemi, F. Khandani, M. Abadyan, Influence of surface effects on size-dependent instability of nano-actuators in the presence of quantum vacuum fluctuations, Phys. Scripta 85 (2012) 035804.

[32] A.W. McFarland, M.A. Poggi, M.J. Doyle, L.A. Bottomley, J.S. Colton, Influence of surface stress on the resonance behavior of microcantilevers, Appl. Phys. Lett. 87 (2005) 053505.

[33] A. Ramezani, A. Alasty, J. Akbari, Influence of van der Waals force on the pull-in parameters of cantilever type nanoscale electrostatic actuators, J. Microsyst. Technol. 12 (2006) 1153-1161.

[34] J. Duan, Z. Li, J. Liu, Pull-in instability analyses for NEMS actuators with quartic shape approximation, Appl. Math. Mech. 37 (2016) $303-314$.

[35] B. Yang, Positive solution for a fourth order boundary value problem, Electron. J. Qual. Theory Differ. Equ. 3 (2005) 1-17.

[36] S. Wolfram, The Mathematica Book, Cambridge University Press, 1996. 